EOMmun Communication et organisation

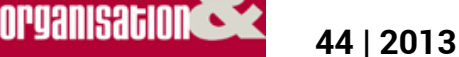

Nouvelles formes de visibilité des individus en entreprise: technologie et temporalité

\title{
Les pratiques vestimentaires en organisation
}

De l'uniformité à la quête de visibilité

\section{Cécilia Brassier-Rodrigues}

\section{OpenEdition \\ Journals}

Édition électronique

URL : http://journals.openedition.org/communicationorganisation/4350

DOI : 10.4000/communicationorganisation.4350

ISSN : $1775-3546$

Éditeur

Presses universitaires de Bordeaux

Édition imprimée

Date de publication : 1 décembre 2013

Pagination : 111-122

ISBN : 978-2-86781-878-3

ISSN : 1168-5549

Référence électronique

Cécilia Brassier-Rodrigues, "Les pratiques vestimentaires en organisation », Communication et organisation [En ligne], 44 | 2013, mis en ligne le 01 décembre 2016, consulté le 21 décembre 2020 URL : http://journals.openedition.org/communicationorganisation/4350 ; DOI : https://doi.org/ 10.4000/communicationorganisation. 4350 


\title{
Les pratiques vestimentaires en organisation de l'uniformité à la quête de visibilité
}

\author{
Cécilia Brossier-Rodrigues'
}

\section{Introduction}

Les organisations sont en mutation. Elles doivent atteindre des objectifs d'efficacité, de productivité, de compétitivité toujours plus élevés, avec pour conséquence une accélération des échanges. Les technologies de l'information et de la communication (TIC) accompagnent ces évolutions. Elles permettent non seulement de s'adapter à la gestion de l'urgence (Pélage Mière, 2006), mais elles sont aussi considérées comme un élément fondamental de l'efficacité des entreprises (Comtet, 2009 ; Guesmi et Rallet, 2012). Dans ce contexte, les contacts interpersonnels deviennent eux aussi davantage numériques grâce notamment aux médias sociaux, à l'utilisation de la messagerie électronique ou encore de l'intranet. Les rencontres physiques sont de plus en plus planifiées, de moins en moins spontanées. Défi, compétition et challenge ont bouleversé l'image de soi en entreprise et sensiblement fait évoluer le sentiment d'appartenance à l'organisation (Boutaud, 1992). B. Massiera (2007), quant à lui, défend l'idée que la culture d'entreprise est en crise, abandonnée. Dans la continuité des travaux de Durkheim, Y. Cusset $(2006,22)$ souligne que le lien social, alors qu'il était traditionnellement « hérité du passé ou imposé par le groupe », serait désormais « de plus en plus construit à partir de l'individu ». Dans un tel environnement d'accélération et d'individualisation de la société, et alors que M. Maffesoli $(1990,29)$ souligne que l'apparence ne se comprend et ne s'envisage que par la présence de l'autre, nous nous demandons quelle importance les acteurs sociaux accordent à l'apparence physique en entreprise dans sa composante vestimentaire. L'usage du numérique a-t-il bouleversé les codes ? La tenue vestimentaire est-elle laissée de côté par les salariés, au profit d'une existence valorisant davantage le «à distance », via la messagerie

1 Cécilia Brassier-Rodrigues est maître de conférences en Sciences de l'Information et de la Communication à Clermont Université. Elle est chercheure au sein de l'équipe Communication et Solidarité (EA 4647). Ses travaux portent sur les enjeux de la communication interpersonnelle dans les organisations. cecilia.brassier@univ-bpclermont.fr 
et les médias sociaux ? Relève-t-elle d'un fait de " costume " (Barthes, 1957), qui contribue à rendre les individus invisibles parce qu'adoptant tous les codes communs à la culture organisationnelle ? Ou alors est-elle un fait « d'habillement » (Barthes, 1957), le vêtement permettant à chacun d'affirmer son identité unique et par là-même de se rendre plus visible aux yeux des autres?

Dans cette recherche, nous nous intéressons à l'usage des pratiques vestimentaires au sein des organisations, alors même que ce sujet a fait l'objet de peu de travaux en sciences de l'information et de la communication (SIC) depuis ceux de R. Barthes (1957). L'apparence physique a souvent été un objet de recherche "négligé, dévalorisé, raillé, ignoré », tant on le considérait comme «frivole, futile » (Amadieu, 2002,10). Le même jugement de valeur est porté au vêtement dont M. Pastoureau $(1995,6)$ rappelait le côté « anecdotique, romantique ou encore esthétique » qu'on lui prête.

Afin d'explorer la manière dont l'habit participe aux nouvelles formes de visibilité en entreprise, nous avons examiné les pratiques vestimentaires des individus dans leur contexte professionnel. Des enquêteurs ont observé 28 individus occupant des fonctions commerciales, pendant cinq jours dans 14 organisations différentes, durant les mois de mai et juin 2013 en Auvergne. Après avoir précisé comment l'enquête a été mise en place, nous analyserons ses résultats. Ils révèlent d'abord une uniformisation des pratiques vestimentaires au sein du groupe professionnel constitué par la population observée. La différenciation entre individus s'opère essentiellement grâce à l'usage d'accessoires, parmi lesquels le téléphone mobile occupe une place à part.

\section{La mise en place de l'enquête par observation}

Dans la continuité des travaux de l'École de Palo Alto (Watzlawick et al., 1972), dont l'une des hypothèses fondamentales est que tout comportement a une valeur communicative, plusieurs études en sociologie et en psychologie ont considéré le vêtement comme un outil de communication identitaire (Delaporte, 1980 ; Lebrun, 2005 ; Gherchanoc et Huet, 2007). La manière dont les individus s'habillent révèle des informations sur leur identité immédiate (qui ils sont) et leur identité désirée (qui ils souhaitent être). L'habit est un vecteur de communication qui contribue à rendre les individus visibles au sein d'un groupe, y compris professionnel.

Louvrage coordonné par Ginette Francequin (2008) illustre la place occupée par le vêtement de travail dans la société française. Dans le contexte professionnel, l'usage du vêtement répond à des contraintes tantôt d'ordre pratique (comme le tablier du boucher), tantôt d'ordre réglementaire (comme l'utilisation de protections obligatoires dans les métiers à risque). Il peut aussi résulter d'une volonté d'apparat ou d'ornementation (comme les vêtements de cérémonie) ou encore " traduire la volonté d'afficher son appartenance 
sociale » (Francequin, 2008, 16). Il peut également correspondre à plusieurs de ces critères. Allant de l'uniforme dans les entreprises de restauration rapide à un style vestimentaire plus décontracté dans les agences de publicité, l'organisation agit plus ou moins ouvertement sur les pratiques vestimentaires de ses employés. Pourtant, dans le contexte organisationnel, l'objet de recherche qu'est le vêtement a été relativement peu analysé.

Barthes $(1957,435)$ propose de distinguer deux aspects dans le vêtement. Le premier est le costume qui relève d'une norme collective et que le sémiologue définit comme " une réalité institutionnelle, essentiellement sociale, indépendante de l'individu, et qui est comme la réserve systématique, normative, dans laquelle il puise sa propre tenue ». Le costume contribuerait à la création de liens entre les individus appartenant à une même " tribu " (Maffesoli, 1990, 28). Dans le cas de l'organisation et plus particulièrement des services qui la composent, cela revient à penser que les pratiques vestimentaires représentent l'un des facteurs définissant le contexte de travail. Selon ces auteurs, pour que la tribu et le vivre ensemble fonctionnent, ces pratiques doivent être codifiées, permettant à chacun de se reconnaître au sein du groupe. Il serait donc nécessaire pour assurer la bonne marche d'un service de définir un costume, auquel devrait se conformer chaque membre de la communauté. Le deuxième aspect dans le vêtement est l'habillement qui relève d'un choix individuel et que Barthes $(1957,435)$ définit comme « une réalité individuelle, véritable acte de vêtement, par lequel l'individu actualise sur lui-même l'institution générale du costume ». C'est ce qui permet à chaque personne d'adapter le costume imposé, de se l'approprier. Dans la réalité, les vêtements portés par les individus résulteraient d'un ajustement entre ces deux dimensions. Le costume tel qu'il est proposé par l'organisation permet aux employés d'avoir confiance en eux, d'obtenir la reconnaissance de leurs pairs, tout en respectant les contraintes liées au métier exercé. L'habillement, quant à lui, est l'élément d'adaptation du costume qui donne l'occasion aux individus de se mettre en scène et de révéler leurs enjeux, par exemple identitaires (donner une certaine image de soi) ou encore relationnels (afficher une proximité avec des personnes d'un groupe). Mais qu'en est-il dans la réalité organisationnelle?

Pour répondre à cette question, nous avons mis en place une enquête qualitative. Des étudiants, de licence et master en commerce international, ont observé pendant cinq jours (une semaine de travail) les pratiques vestimentaires de deux employés, chacun appartenant au service commercial au sein duquel ils réalisaient leur stage depuis plusieurs semaines. Le choix des sujets d'observation s'est fait en respectant deux consignes. D'abord, il était primordial que l'enquêteur puisse voir quotidiennement les personnes observées. Ensuite, les deux individus devaient avoir des rôles et des statuts différents au sein du service. Avant de commencer la collecte des données, les enquêteurs ont également renseigné un certain nombre d'informations 
sur le service de l'entreprise dont dépendaient les personnes qui allaient être observées : taille, niveaux hiérarchiques présents, type de relations de travail, style vestimentaire adopté, etc. Il s'agissait de mieux comprendre le contexte dans lequel les sujets évoluaient quotidiennement. Au final, nous avons collecté des données dans 14 organisations différentes sur le plan de la taille, du secteur d'activité.

Les observations que nous avons faites avaient pour objectif d'explorer la manière dont l'habit participe aux nouvelles formes de visibilité en entreprise, tout en vérifiant plusieurs hypothèses. Tout d'abord, nous avons mentionné que le mode de fonctionnement des organisations avait évolué, favorisant la multiplication des échanges à distance entre individus. C'est particulièrement le cas dans les métiers liés au commerce où les contacts avec les clients et les fournisseurs font partie des activités quotidiennes, souvent via les TIC. Ce faisant, nous nous demandons si l'utilisation accrue des TIC a un impact sur l'apparence physique des individus. Prêtent-ils moins d'attention à leur tenue vestimentaire, puisque les occasions de contact physique sont moins nombreuses ? Ensuite, nous souhaitons analyser de quelle manière les pratiques vestimentaires renvoient à un fait de costume et/ou à un fait d'habillement (Barthes, 1957). À cette fin, les enquêteurs ont d'abord décrit le style vestimentaire adopté par l'ensemble du service, révélant l'existence (ou pas) d'une pratique uniforme. Ensuite, l'observation de deux sujets de statut différent au sein de chaque service a permis d'étudier comment chacun adoptait et adaptait les normes du groupe, lorsqu'elles existaient.

\section{Des pratiques vestimentaires uniformes dans la sphère professionnelle}

Le vêtement de travail des hommes occupant des fonctions commerciales ${ }^{2}$ dans les organisations observées est un pantalon associé à une chemise. Les femmes ont un choix plus large, elles portent tantôt un pantalon ou une jupe avec un chemisier ou un pull, tantôt une robe. On a noté que lorsque les personnes observées avaient des rendez-vous à l'extérieur (avec des clients, des fournisseurs) ou des réunions dans d'autres services de l'entreprise, elles modifiaient leurs pratiques : usage de la veste de costume et de la cravate (moins souvent cependant) pour les hommes, port d'un pantalon noir et d'une veste de tailleur pour les femmes. Les résultats de l'enquête montrent en outre qu'il existe des usages vestimentaires relativement similaires entre les entreprises observées. Les différences s'établissent au niveau du type de pantalon porté (des jeans en majorité ou des pantalons à pinces, tant pour les hommes que pour les femmes) ou encore du choix des couleurs (certains hommes osent les chemises à fleur et des femmes les couleurs vives, tandis que dans d'autres organisations les coloris sont moins variés et plus sobres). Ces pratiques suggèrent la présence toujours réelle d'une culture d'entreprise,

2 Les fonctions observées sont les suivantes : directeur commercial, responsable de secteur, responsable de zone, assistant export. 
confirmant que les individus, par leurs choix vestimentaires, se conforment aux normes proposées par l'entreprise et se mettent ainsi en cohérence avec les valeurs qu'elle véhicule (Greenan et al. 2005). Cependant, les similitudes entre organisations étant fortes, il semble exister un costume de commercial, porté par les personnes appartenant à ce groupe professionnel.

Les résultats de l'enquête laissent donc apparaître une certaine uniformité, mais également une relative décontraction dans le choix de la tenue vestimentaire des employés occupant des fonctions commerciales. Amadieu avait déjà fait ce constat de souplesse dès 2002 (122). Les TIC n'en sont pas à l'origine, puisqu'alors elles n'occupaient pas encore une place prédominante dans l'environnement de travail. En revanche, les pratiques constatées aujourd'hui conduisent à penser qu'elles ont intensifié cette tendance grâce à la multiplication des contacts à distance. Dans ce contexte de dématérialisation des échanges, les occasions de rendez-vous physique avec des personnes extérieures à l'entreprise sont, en conséquence, moins nombreuses. Cependant, dès qu'elles se produisent, les résultats de l'enquête montrent que les employés recherchent une plus grande rigueur vestimentaire.

En somme, les commerciaux adoptent les normes vestimentaires que le groupe, auquel ils appartiennent à un moment donné, définit pour être reconnus comme l'un de ses membres. Lorsqu'ils sont au sein de leur équipe, ils se laissent aller à une relative souplesse. Lorsqu'ils la quittent et vont à la rencontre de partenaires (clients, fournisseurs, autre personne de l'entreprise), ils sont plus rigoureux dans leur choix vestimentaire. À ce moment-là, ils endossent le costume de commercial adapté à la tenue de leur rôle et plus conforme aux attentes de leur groupe professionnel. Cette fonction de reconnaissance de l'habit existe depuis longtemps. Ainsi, le vêtement a d'abord été analysé sous l'angle du «triangle des motivations » (Burgelin, 1996, 89) : protection, pudeur, parure. Selon les chercheurs, l'homme se serait habillé afin de protéger son corps des conditions climatiques extérieures, de l'embellir et d'accomplir ses tâches quotidiennes (Delaporte, 1980 ; Pastoureau, 1995). Puis, Barthes (1957) a ajouté à cette liste la fonction sociologique, soulignant que les vêtements constituent des signes (Delaporte, 1980), qui contribuent à légitimer celui qui parle (Bourdieu, 2001), à le rendre identifiable, visible aux yeux des autres. Ils améliorent la compréhension des rôles tenus par les acteurs. Outre cette dimension signifiante, la fonction sociologique présente également une composante symbolique : le port du vêtement permet d'afficher une appartenance. Ce n'est pas tant l'habit, assemblage de plusieurs pièces de tissus, que son choix, son usage et ses implications qui sont analysés. Tous ces facteurs apportent du sens à la communication des acteurs. Le vêtement porte intrinsèquement un sens que chacun peut lire. D'un simple regard, il permet d'apprécier des éléments d'identité d'un individu ; il permet de créer un a priori positif (ou négatif), un sentiment d'appartenance (ou de rejet) et donc de susciter l'envie d'entrer en interaction (ou pas). En ce sens, les habits 
sont « des outils de communication identitaires qui servent à afficher un statut social, politique ou religieux, ainsi qu'à connoter des manières d'être » (Gherchanoc et Huet, 2007, 13).

Dans le contexte organisationnel, les salariés semblent donc être en quête de reconnaissance, entendue au sens de « critère pour penser la normalité d'une société qui offre à ses membres les conditions de pouvoir se réaliser » (Heller, 2009, 115). Grâce à l'habit, les acteurs organisationnels rendent visibles des caractéristiques qui permettent aux membres du groupe de les identifier et de les admettre. Complétant la définition de la reconnaissance de l'individu au travail d'Andonova et Vacher $(2009,137)$, le vêtement permettrait à l'individu d'opérer une reconnaissance identitaire, première étape nécessaire pour être admis dans un groupe, avant d'aller vers une forme plus aboutie de partage et d'échange. Dans le même temps, le fait d'endosser un costume identique rend les individus invisibles aux yeux des autres, car ils sont jugés peu différents les uns des autres. A. Monjaret (2011) avait déjà fait état de cette invisibilité lors d'une étude réalisée sur le vêtement de travail du personnel technique dans les hôpitaux, allant même jusqu'à parler de «transparence » (105).

Face à autant d'uniformisation, la volonté de différenciation soulignée par le fait d'habillement trouve elle aussi sa place dans le contexte organisationnel. Selon les résultats de notre étude, pour exprimer leur personnalité, pour se démarquer des autres à l'intérieur du groupe, les individus utilisent des accessoires.

\section{La contribution des accessoires vestimentaires à la visibilité des acteurs organisationnels}

$\mathrm{Au}$ sein de la fonction sociologique et identitaire, le vêtement offre une possibilité de différenciation (Pop, 1984, 50). Elle peut être spatiale, lorsque les individus choisissent leur tenue en fonction de la région dans laquelle ils vivent. Elle peut être temporelle, lorsque le choix des vêtements est influencé par l'âge ou par l'époque dans laquelle on vit. La différenciation peut aussi être sociale, puisque le vêtement donne des informations sur le statut social de celui qui le porte ${ }^{3}$. Au sein d'un groupe déjà constitué et dont les usages vestimentaires sont relativement uniformes, les accessoires peuvent servir cet objectif de différenciation de deux manières : soit l'individu est le seul à porter un accessoire particulier (c'est le cas de certains accessoires traditionnels comme le port du chapeau pour un homme), soit les caractéristiques de l'objet sont différentes de celles choisies par les autres porteurs (en raison de la marque, de la couleur, etc.).

L'accessoire est un objet qui accompagne une chose principale, qui s'ajoute à titre secondaire. Lorsqu'il est vestimentaire, il existe en complément du vêtement et ne peut pas être porté seul (une ceinture est associée au port

3 L'exposition « Costumer le pouvoir », mise en scène au Centre National du Costume de Scène de Moulins, de janvier à mai 2013, a illustré ce point. 
d'un pantalon ou d'une jupe). Il remplit les mêmes fonctions que l'habit - protection, ornementation, symbolique et identitaire - et il peut les cumuler. Par exemple, un sac à main permet non seulement de protéger les objets qu'il abrite, mais également d'embellir la tenue et d'afficher une appartenance sociale. Lors des observations réalisées, nous avons constaté que les individus utilisaient deux catégories d'accessoires : traditionnel et électronique. Ainsi, parmi les accessoires traditionnels, figurent la cravate, la ceinture, la montre ou encore les lunettes de soleil pour les hommes. Les femmes, elles, adjoignent volontiers à leur tenue un foulard, des bijoux (collier, bagues, boucles d'oreilles), des lunettes, des objets pour les cheveux, un sac à main. Ce sont là des accessoires classiquement liés au port du vêtement. En revanche, l'association d'accessoires électroniques à l'habit est originale. Pourtant, lorsque les enquêteurs ont décrit la tenue des personnes observées, ils ont fréquemment noté la possession d'un téléphone mobile, qu'ils ont confondu dès lors avec un accessoire vestimentaire.

De nombreux travaux ont pris pour objet d'étude le téléphone mobile ces dernières années tant dans le domaine des SIC, de la sociologie, de l'économie (Jaureguiberry, 2003 ; Gaglio, 2008 ; Licoppe et Zouinar, 2009). Comme le confirment les enquêtes menées régulièrement par l'Observatoire sociétal du téléphone mobile ${ }^{4}$, il occupe une place croissante dans la vie des Français et ses usages se multiplient. Il est considéré par certains chercheurs comme un outil de développement du web relationnel dans les organisations (Guesmi et Rallet, 2012). Il participerait, à ce titre, à la reconfiguration des genres communicationnels. Pour d'autres, "le téléphone mobile permettrait de remplir les vides et de rationnaliser l'ensemble des moments de vie » (Godard, 2007, 34). Ce faisant, les chercheurs s'interrogent sur la manière dont le téléphone mobile rend plus perméables les frontières entre la sphère publique et la sphère privée (Le Douarin, 2007 ; Boboc et Metzger, 2009).

En revanche, le téléphone mobile n'avait pas encore été considéré comme un élément d'habillement des individus, à notre connaissance. Au-delà des observations réalisées, il convient désormais de s'interroger sur le statut de cet accessoire : est-il utilisé comme un objet d'ornementation, affiche-t-il une appartenance ? Pourquoi les usagers le montrent-ils alors même qu'il peut rester au fond d'une poche ou d'un sac à main ? Peut-on faire le lien avec une manifestation de la « disponibilité permanente » de l'individu (Godard, 2007, 35 ), puisque celui-ci affiche qu'il est joignable immédiatement et en tout lieu par l'ensemble de ses relations? Il révélerait alors son appartenance à plusieurs communautés en dehors de l'organisation et sa connexion permanente à celles-ci dans le contexte professionnel, posant encore une fois la question de la frontière entre sphères publique et privée. Afin de compléter les résultats

4 « Sixième édition de l'Observatoire sociétal du téléphone mobile », décembre 2010, http://www.fftelecoms. $\mathrm{org} /$ thematiques/usages-et-contenus/observatoire-societal 
obtenus par les observations, nous réaliserons des entretiens avec les usagers dans une prochaine étude.

En somme, il semble que les accessoires, qu'ils soient traditionnels ou électroniques, contribuent à une meilleure visibilité des individus au sein du groupe, alors que ceux-ci adoptent par ailleurs des pratiques vestimentaires uniformes. Il s'agit d'éléments qui permettent à chacun de satisfaire « tant le besoin d'appartenance et d'identité qui soudait l'unité du groupe, que le désir individuel et légitime de se particulariser, de se différencier des siens " (Pop, 1984, 50). Ce sont aussi des objets qui permettraient d'afficher l'appartenance à différents groupes, en dehors de l'organisation. Par exemple, porter une écharpe jaune et bleue en Auvergne fait immédiatement d'un individu un supporter du club de rugby régional.

\section{Conclusion}

Que ce soit dans la société civile ou au sein des organisations, les individus se rencontrent de moins en moins en face à face, privilégiant des formes de contact plus rapides, spontanées, numériques (Cusset, 2006). Dans ce contexte, alors même que le vêtement est un facteur important de création de lien social « de participation organique »(Paugam, 2009, 71), de visibilité et de reconnaissance des individus, nous nous demandons comment il est abordé dans les organisations aujourd'hui. Est-il « un fait de costume » qui rend l'ensemble des membres du groupe invisibles (Barthes, 1957) ? Est-il « un fait d'habillement » qui prend en compte les caractéristiques uniques de chaque membre du groupe, permettant d'exprimer l'individualité de chacun (Barthes, 1957) ? Comment s'opère aujourd'hui l'ajustement entre ces deux dimensions?

En prenant pour objet d'étude le vêtement, symbole de l'apparence et signe de distinction des acteurs au sein des organisations, nous avons exploré la manière dont l'habit participe aux nouvelles formes de visibilité en entreprise, dans un contexte d'omniprésence du numérique. Nos résultats montrent que la nécessité de travailler ensemble paraît inciter les individus à adopter un style vestimentaire commun, premier élément visible de l'existence de codes partagés et de reconnaissance des individus entre eux. Le fait de costume est ainsi avéré et un uniforme se dessine. Cette pratique a pour conséquence de rendre les individus invisibles au sein du groupe, puisque tous respectent les mêmes codes vestimentaires. Pourtant, le souhait de mettre en avant son individualité existe et il se formalise par l'utilisation d'accessoires. Par leur diversité, ceux-ci peuvent souligner de manière très personnelle les vêtements portés par les individus et ainsi participer au fait d'habillement. L'accessoire rend chaque individu visible aux yeux des autres, en affichant son libre arbitre, sa capacité à associer des éléments au costume imposé. À ce stade, le téléphone mobile est apparu comme un accessoire vestimentaire. Il illustre aussi l'importance du numérique dans le quotidien des individus, qui 
choisissent d'afficher cet objet leur offrant une connexion permanente avec les autres membres du groupe et avec leurs proches.

Malgré leurs limites, qui tiennent notamment au fait que seule une étude qualitative exploratoire a été réalisée, nos résultats suggèrent que le vêtement et ses accessoires participent à la visibilité et à la reconnaissance des individus en entreprise. De même, ils révèlent que la question de l'entrelacement des sphères publique et privée se pose aussi dans le contexte vestimentaire.

En définitive, l'introduction des TIC dans l'organisation n'a pas fondamentalement bouleversé les codes vestimentaires. Elle a toutefois contribué à modifier les pratiques. D'une part, elle a favorisé une plus grande souplesse dans les usages grâce à la multiplication des contacts à distance. D'autre part, elle a conduit à l'apparition du téléphone mobile dans les accessoires vestimentaires, faisant de celui-ci un objet de visibilité. Cette étude mériterait d'être complétée par des entretiens en face-à-face qui permettraient de préciser le rôle que les usagers attribuent au téléphone mobile comme accessoire vestimentaire.

\section{BIBLIOGRAPHIE}

AMADIEU J. F., Le poids des apparences. Paris : Odile Jacob, 2002, 194 p.

ANDONOVA Y., VACHER B., "Visibilité et reconnaissance de l'individu au travail », in Communication E Organisation, 2009, n 36, p. 136-147.

BARTHES R., "Histoire et sociologie du vêtement », in Annales Economie, Société, Civilisations, 1957, n 3, p. 430-441.

BOBOC A. et METZGER J. L., « Du privé vers le professionnel, une dynamique des apprentissages croisés autour des TIC », in Savoirs, 2009, n² 2, p. 158-179.

BOURDIEU P., Langage et pouvoir symbolique. Paris : Seuil, 2001, 423 p.

BOUTAUD J. J., «Image de soi et organisation », in Communication E Organisation, 1992, n $^{\circ} 1$, p. $2-12$.

BURGELIN O., « Barthes et le vêtement », in Communications, 1996, n 63, p. 81100.

COMTET I., Entre usage professionnel des TIC et structure organisationnelle : la capacité au bricolage comme compétence adaptative, in Études de communication, 2009, $\mathrm{n}^{\circ} 33$, p. 119-134.

CUSSET Y., Les évolutions du lien social, Un état des lieux, in Horizons Stratégiques, 2006, n 2, p. 21-36.

DELAPORTE Y., Le signe vestimentaire, in L'Homme, 1980, vol. 20, n 3, p. 109142.

FRANCEQUIN G., Le vêtement de travail, une deuxième peau. Paris : Erès, 2008, $276 \mathrm{p}$.

GHERCHANOC F., HUET V., Pratiques politiques et culturelles du vêtement, in Revue Historique, 2007, n 641, p. 3-30. 
GAGLIO G., « La dynamique des normes de consommation : le cas de l'avènement de la téléphonie mobile en France ", in Revue Française de Socio-Économie, 2008, vol. 2, p. 181-198.

GODARD F., "Vie publique et vie privée : de nouveaux régimes temporels ", in Réseaux, 2007, n 140, p. 29-65.

GREENAN N. et al., " Enquête changements organisationnels et informatisation ", in Réseaux, 2005, n 134, p. 21-63.

GUESMI S. et RALLET A. WEB 2.0 et outil de coordination décentralisée, in Revue Française de Gestion, 2012, n 224, p. 139-151.

HELLER T., « Reconnaissance et communication : une logique de l'assujettissement », in Communication E Organisation, 2009, n 36, p. 108-120.

JAUREGUIBERRY F., Les branchés du portable. Paris : PUF, 2003, 196 p.

LEBRUN C., "Fonctions métaphoriques et métonymiques du vêtement », in L'Esprit du Temps, 2005, n 53, p. 613-626.

LE DOUARIN L., " Les chemins de l'articulation entre vie privée et vie professionnelle », in Réseaux, 2007, n 140, p. 101-132.

LICOPPE C., ZOUINAR M., " Présentation du dossier : Les usages avancés du téléphone mobile », in Réseaux, 2009, vol. 156, p. 9-12.

MAFFESOLI M., Au creux des apparences. Pour une éthique de l'esthétique. Paris : La Table Ronde, 2007, 316 p.

MASSIERA B., "Culture d'entreprise, l'échec d'un concept ", in Communication, 2007, vol. 25, n 2, p. 131-147.

MONJARET A., «Du bleu de chauffe au jean : Les jeux de l'apparence des “ouvriers” à l'hôpital, entre traditions corporatistes et normes institutionnelles renouvelées ", in Sociologie et sociétés, 2011, vol. 43, n 1, p. 99-124.

PASTOUREAU M., "Pratiques et symboliques vestimentaires », in Médiévales, 1995, n² 29, p. 5-7.

PAUGAM S., Le lien social. Paris : PUF, 2009, 127 p.

PELAGE MIERE T., "Les TIC dans l'entreprise : entre urgence et réactivité », in Communication E Organisation, 2006, n 29, p. 196-205.

POP D., «Évolution d'un système vestimentaire dans les sociétés rurales de Roumanie ", in L'Homme, 1984, tome 24, n 1, p. 43-64.

WATZLAWICK P., BEAVINJ.D.,JACKSON D. D., Une logique de la communication. Paris : Le Seuil, 1972, 280 p.

Résumé : Dans cette recherche, nous prenons pour objet d'étude le vêtement, symbole de l'apparence et signe de distinction des acteurs au sein des organisations. Nous proposons d'explorer la manière dont l'habit participe aux nouvelles formes de visibilité en entreprise, dans un contexte d'omniprésence du numérique. Les résultats d'une observation, réalisée en mai et juin 2013 auprès de 28 personnes travaillant dans des fonctions commerciales, indiquent que le vêtement est utilisé par l'individu à des fins de reconnaissance et de respect du groupe au sein duquel il évolue, en vue de faciliter son adhésion. Ils montrent également que l'individualité s'exprime au moyen 
d'accessoires, parmi lesquels figure le téléphone mobile. Cet état de fait contribuerait à affirmer l'unicité de chacun, révélant l'importance toujours réelle de la fonction de parure et d'ornementation associée au vêtement.

Mots-clés: apparence physique, pratiques vestimentaires, contexte organisationnel, visibilité.

Abstract: This investigation deals with clothes, as they are a symbol of appearance and a sign of distinction among individuals inside organizations. In a context dominated by digital technology, we propose to analyse how clothing participates in the new forms of visibility inside firms. An observation has been realized, in May and June 2013, on 28 individuals occupying commercial jobs at the international level. The results reveal that clothes are used by people in order to be recognized and respected by the group they belong to. They also show that people use accessories to express their individuality, among those we find the mobile phone.

Keywords: physical appearance, clothing practices, organizational context, visibility. 
